Article

\title{
Development of Sidewalk Block Pavement Condition Index (SBPCI) using Analytical Hierarchy Process
}

\author{
Jangrak Kim ${ }^{1}$, Daegeun Park ${ }^{2}$, Youngchan Suh ${ }^{1}$ and Donghyuk Jung ${ }^{3, * \mathbb{C}}$ \\ 1 Department of Transportation and Logistics Engineering, Hanyang University, 55 Hanyangdaehak-ro, \\ Sangnok-gu, Ansan-si, Gyeonggi-do 15588, Korea; jaksal93@hanyang.ac.kr (J.K.); suhyc@hanyang.ac.kr (Y.S.) \\ 2 Seoul Institute of Technology, 8F Maebongsan-ro 37, Mapo-gu, Seoul 03909, Korea; dgpark@sit.re.kr \\ 3 Korea Institute of Civil Engineering and Building Technology, (Daehwa-Dong)283, Goyangdae-Ro, \\ Ilsanseo-Gu, Goyang-Si, Gyeonggi-do 10223, Korea \\ * Correspondence: jdh88@kict.re.kr
}

Received: 25 September 2019; Accepted: 9 December 2019; Published: 11 December 2019

\begin{abstract}
This study aimed at developing SBPCI (Sidewalk Block Pavement Condition Index) with sidewalk pavement condition survey data of Seoul city in order to attain a quantitative evaluation method of sidewalk pavement condition. The distress patterns of sidewalk pavement were classified into four groups of Crack/Loss, Roughness, Aging, and Corner Break. AHP (Analytic Hierarchy Process) technique was employed on the basis of the raking process of 31 pavement managers in order to analyze the influence of the distress patterns on the sidewalk pavement condition. The AHP analysis result indicated the weight of pop out, roughness, surface abrasion, and corner break were $0.521,0.244,0.164$, and 0.070 , respectively, by distress type. A model equation was derived by using the sidewalk pavement condition data from 420 sections. The correlation analysis between the result of the model equation and distress type revealed that the correlation of corner break was low to be excluded from SBPCI model; while pop out, roughness, and surface abrasion were statistically significant to be used as three variables of the developed SBPCI model.
\end{abstract}

Keywords: sidewalk pavement; block pavement; pavement condition index; pavement management; AHP; sensitivity analysis

\section{Introduction}

Modern society achieved rapid civilization development owing to fast industrialization starting from the 18th century. However, its mechanization brought problems of shortened labor hours and physical activity of humans. Such green transportation methods such as public transportation, cycling, walking, etc., as alternatives to automobiles, have actively been advocated in order to deal with the above problems. Among those methods, the interest in sidewalk walking has been continuously growing.

Efficient management of sidewalk pavement is necessary in order to ensure a safe and pleasant environment for pedestrians. Starting with the Pavement Management Conference held at Toronto, Canada in the 1980s [1], studies of PMS (Pavement Management System) have been continuously carried out. Prioritization of an objective assessment of pavement conditions of extensive sidewalks should be performed in order to maintain the sidewalk pavement in good condition. The result should be reflected in setting a budget for sidewalk pavement construction. Nonetheless, it is current reality that the manpower and budget for the sidewalk management are lacking, and, especially, efficient sidewalk management method is also unprepared.

Additionally, the current situation is such that maintenance and management of sidewalk pavement is not adequate with the sidewalk pavement data from PMS alone. Especially, since municipalities of the Republic of Korea have not established the standard for quantization of sidewalk 
pavement condition, survey method, and detail repair standard; therefore, the implementation of efficient sidewalk pavement management system is more necessary.

Although the condition indices applied to airports, expressways, and national roads have relatively been proposed in variety and have been used, the survey items and evaluation method of sidewalk pavement condition are very different from each other so that it is difficult to apply them as they are. Moreover, the result of reviewing overseas cases indicated that it was difficult to find a case of comprehensive evaluation index in consideration of complex distress types of sidewalk pavement, while the repair standard for a specific sidewalk pavement distress was established.

Accordingly, this study aimed at carrying out an investigation of systematic and quantitative maintenance and management method of sidewalk pavement to develop a SBPCI (Sidewalk Block Pavement Condition Index) by using AHP.

This study targeted 420 deteriorated sidewalk pavement sections among routes of Seoul city for the quantification of sidewalk pavement condition. Surface images were taken and used for the analysis of sidewalk pavement, and a database was built by classifying pavement status, presence or absence of a bicycle road, status of vehicle entrance and exit, and distress status. Additionally, AHP (Analytic Hierarchy Process) based on the responses of sidewalk pavement management officers was used to compute the weight of each distress type in order to analyze the influence of distress types on pavement condition. Then, the weight was used to develop SBPCI.

\section{Literature Review}

\subsection{Status of Sidewalk Pavement Condition Management by Country}

TIMMS (Transportation Infrastructure Maintenance Management System) including sidewalk pavement has been developed in order to minimize maintenance/serviceability and resident complaints of infrastructures in Utah, USA [2]. The city of Oakland, California, carries out sidewalk pavement management in consideration of ADA (Americans with Disabilities Act), and the distress types have been largely classified into step, crack, uplift, and settlement to perform the survey of the pavement condition. The surveyed data are linked with GIS (Geographic Information System) to build a database of the images of deteriorated pavement sections, which in turn is used to establish a database for the selection of the maintenance/management target section and repair budget, etc. [3].

The city of Palermo, Italy, defined a pedestrian safety index in consideration of such variables as the sidewalk width, sidewalk pavement condition, location of obstacle fixtures, etc. [4]. The city of Trieste, Italy, developed SCI (Sidewalk Condition Index) for quantitative evaluation of pedestrian safety and sidewalk pavement condition, and collected data through questionnaires from local residents and visual surveys of the sidewalk pavement condition. The survey was principally carried out targeting the paved road to establish a strategy for the maintenance and management of sidewalk pavement through SCI [5].

London, United Kingdom, is collecting data on the pavement sections in need of repair through "Highway Asset Management Survey-Footways". The pavement condition survey items were classified into asphalt (abrasion, crack, settlement), concrete (crack, unevenness, crack and unevenness, puddle, around manhole), and block pavement (crack, unevenness, crack and unevenness) to apply the distress types differentially by the pavement types. Moreover, the sidewalks have been classified into four grades, depending on the importance, to propose a survey period, and repair time by the grade of the sidewalk has been proposed [6].

\subsection{Using AHP in Pavement Management}

Ahmed et al. [7] used AHP to determine the maintenance/repair priority based on distress level of the sidewalk pavement and carried out a comparison study with existing RCI (Road Condition Index) by using the AHP result. The correlation analysis of each result of AHP, RCI and priority indicated 
$79 \%$ correlation for the case of the AHP and RCI result, and $85 \%$ correlation for the case of priority, thus showing a satisfactory result.

Li et al. [8] also used AHP in order to determine the maintenance/repair priority depending on the road pavement condition at network level. A total of five factors (road grade, pavement condition, strength, ESALs, age) were used to determine the priority. Pavement condition was found to be the most sensitive variable of influence on the determination of maintenance/repair priority among those factors.

While the preceding researches regarding maintenance decision-making on sidewalk pavement has so far been limited to visual survey and qualitative assessments, this study has been conducted with the aim of enhancing the efficiency of the existing sidewalk pavement maintenance system by presenting a new quantitative index. In addition, we went through in-depth discussions with working level staff members in order to apply the existing system conveniently without significantly changing the current system. To do so, this paper uses AHP in order to reflect the opinions of the working staff members.

\section{Methodology}

\subsection{Data Collection}

Data for computation of sidewalk block pavement condition index (SBPCI) was separately collected depending on the condition of the sidewalk pavement. Since the data of the sidewalk construction area and deteriorated area should be collected in order to quantitatively assess the condition of the sidewalk pavement, the construction areas of sidewalk pavement were computed by using an index map and giving an ID for each section as shown in Figure 1. The deteriorated area of the sidewalk was directly investigated for the distress type and distress amount by the surveyors, who identified the location and ID through the digital map. Additionally, the basic data of sidewalk (type of sidewalk pavement, pattern, etc.), which constitute the foundation for building the data, were also simultaneously surveyed.

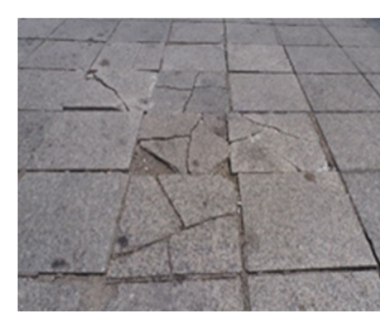

(a)

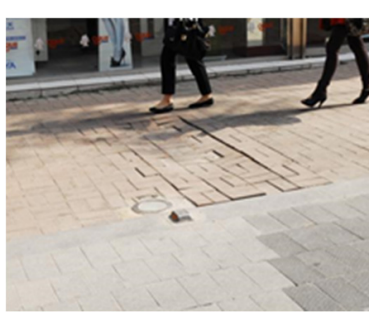

(b)

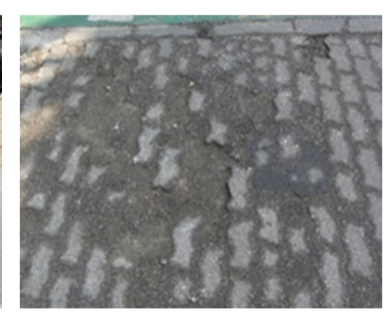

(c)

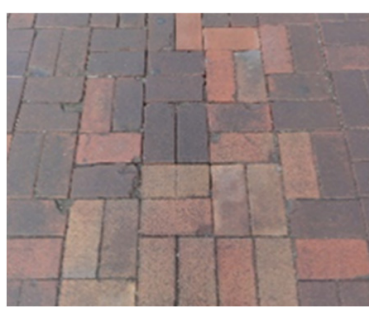

(d)

Figure 1. Type of sidewalk block pavement's distress. (a) Pop out, (b) Roughness, (c) Surface abrasion, and (d) Corner break.

\subsection{Definition of Distress Types of Sidewalk Pavement}

A Field investigation was carried out with maintenance and management experts in order to find out the deterioration forms of the sidewalk. The survey results indicated four distress types occurring on sidewalk pavements. These types included crack or detachment of the sidewalk block (Po: Pop out), roughness such as step, unevenness, settlement, uplift, puddling, etc., (Ro: Roughness), abrasion of block surface (Sa: Surface abrasion), and Break-off of block corner (Cb: Corner break). Most observed distress types belonged to one of the above four types, and Table 1 below defines and summarizes the distress forms in detail, and causes of the distress by each distress type. Figure 2 represents a typical example of each distress type. 
Table 1. Definitions of Sidewalk Block Pavement Distress Types.

\begin{tabular}{|c|c|c|c|c|}
\hline Classification & Pop out & Roughness & Surface abrasion & Corner break \\
\hline Distress Type & $\begin{array}{l}\text { - Block Crack } \\
\text { - Block Pop out }\end{array}$ & $\begin{array}{l}\text { - Partial subsidence } \\
\text { - Water Gouge } \\
\text { - Bumpy Road } \\
\text { - Joint widening }\end{array}$ & $\begin{array}{c}\text { - Abrasion } \\
\text { - Surface desquamation }\end{array}$ & - Corner Break \\
\hline $\begin{array}{l}\text { Cause of } \\
\text { Distress }\end{array}$ & $\begin{array}{l}\text { - Poor Strength } \\
\text { - Lack of bearing capacity } \\
\text { - Vehicle Passage }\end{array}$ & $\begin{array}{c}\text { - Poor Stable Layer } \\
\text { - Structural Bump } \\
\text { - Lack of bearing capacity }\end{array}$ & $\begin{array}{l}\text { - Poor materials } \\
\text { - Long-term service }\end{array}$ & $\begin{array}{l}\text { - Joint Sand sweeped } \\
\text { - Poor construction } \\
\text { - Poor Strength }\end{array}$ \\
\hline
\end{tabular}

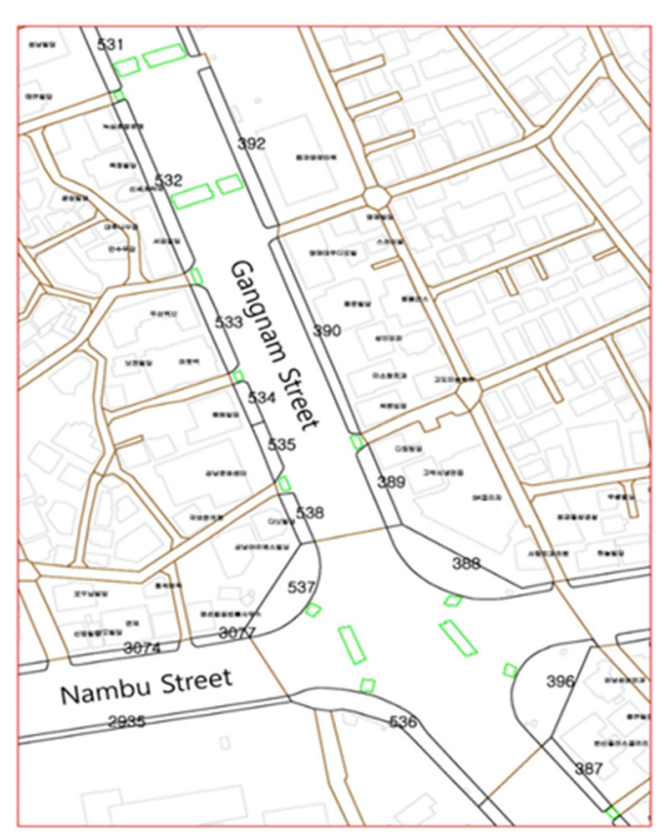

(a) Digital Map

\begin{tabular}{|c|c|c|c|c|c|c|}
\hline \multicolumn{7}{|c|}{ o Location Information } \\
\hline $\begin{array}{l}\text { Road } \\
\text { name }\end{array}$ & \multicolumn{2}{|c|}{$\begin{array}{l}\text { Gangnam } \\
\text { Street }\end{array}$} & ID & 389 & Date & 20150605 \\
\hline Start & \multicolumn{2}{|c|}{388} & End & 390 & & \\
\hline \multicolumn{7}{|c|}{ - Sidewalk Information } \\
\hline Material & \multicolumn{2}{|c|}{$\begin{array}{l}\text { concrete } \\
\text { block }\end{array}$} & $\begin{array}{l}\text { block } \\
\text { size }\end{array}$ & \multicolumn{3}{|c|}{$100 \times 200(\mathrm{~mm})$} \\
\hline Color & \multicolumn{2}{|c|}{ Brown } & $\begin{array}{l}\text { bicycle } \\
\text { path }\end{array}$ & \multicolumn{3}{|c|}{ Yes / No } \\
\hline \multicolumn{7}{|l|}{ Shape } \\
\hline \multicolumn{7}{|c|}{$\circ$ Distress } \\
\hline \multicolumn{2}{|l|}{ Type } & \multicolumn{2}{|c|}{ Area $(\mathrm{m} \times \mathrm{m})$} & \multicolumn{2}{|c|}{ Photo ID } & Location \\
\hline \multicolumn{2}{|c|}{ Roughness } & \multicolumn{2}{|c|}{$4.5 \times 3.0$} & \multicolumn{2}{|c|}{0003,0004} & 1 \\
\hline \multicolumn{2}{|l|}{ Pop out } & \multicolumn{2}{|c|}{$1.5 \times 1.0$} & \multicolumn{2}{|c|}{0005,0006} & 2 \\
\hline
\end{tabular}

(b) Investigation Sheet

Figure 2. Example of digital map and investigation sheet. (a) Digital Map, and (b) Investigation Sheet.

\subsection{Measurement of Deteriorated Area}

The distress types were classified in order to measure the deteriorated area of the sidewalk pavement by assuming a rectangular shape of the deteriorated area. The minimum area of distress was set to be $1 \mathrm{~m}^{2}$ in consideration of the minimum area of maintenance/repair.

\subsection{Computation of Distress Rate}

The amount of distress occurring at a unit section of the sidewalk was computed for each distress type and made into a database. The distress amount of each distress type was computed by the following method (Equation (1)):

$$
\operatorname{Dij}(\%)=\frac{(D i A) j}{T A j} \times 100
$$

where, $D i=$ Rate of Distress, $i$ in Section $j(\%) ;(D i A) j=$ Area of Distress, $i$ in Section $j\left(\mathrm{~m}^{\wedge} 2\right) ; T A j=$ Total Area (m^2); $i=$ Pop-out, Roughness, Surface abrasion, Corner break; $j=$ Section.

\section{Analytical Hierarchy Process Models}

AHP (Analytic Hierarchy Process) classifies various factors in a hierarchy to find out the importance of each property so as to suggest priority of each alternative. This study employed AHP in order to analyze the level of influence of the four distress types of sidewalk pavement on the pavement 
condition. A questionnaire survey was carried out to obtain responses from 31 sidewalk management civil service officers.

Prior to the AHP analysis, responses from the 31 officers to the questionnaire "Quantitative Rate of Determinants of Sidewalk Pavement Repair Section Selection" were gathered. The sum of the rates of the six determinants of pavement condition, urban aesthetic view, route grade, civil complaints, pavement material, and other, was set to $100 \%$, and the survey result of rates of each determinant is shown in the following Table 2. It shows that 'pavement condition' of $76.8 \%$ was the most important factor of influence. It also attests to the validity of applying only sidewalk pavement distress to the development of SBPCI evaluation model in this study.

Table 2. Survey of Rate of Determinants of Selecting Sidewalk Pavement Section for Repair.

\begin{tabular}{cccccccc}
\hline Classification & $\begin{array}{c}\text { Pavement } \\
\text { Condition }\end{array}$ & $\begin{array}{c}\text { Urban } \\
\text { Esthetic View }\end{array}$ & $\begin{array}{c}\text { Route } \\
\text { Grade }\end{array}$ & $\begin{array}{c}\text { Civil } \\
\text { Complaints }\end{array}$ & $\begin{array}{c}\text { Pavement } \\
\text { Material }\end{array}$ & Other & Sum \\
\hline Rate & $76.8 \%$ & $5.4 \%$ & $5.1 \%$ & $7.5 \%$ & $3.4 \%$ & $1.8 \%$ & $100.0 \%$ \\
\hline
\end{tabular}

\subsection{Hierarchy Structure}

A hierarchy structure was built based on the distress types of sidewalk pavement. In addition, the major distresses that could affect the condition of the sidewalk pavement is carried out in the questionnaire above Table 2. The derived distress types are shown in the following Figure 3.

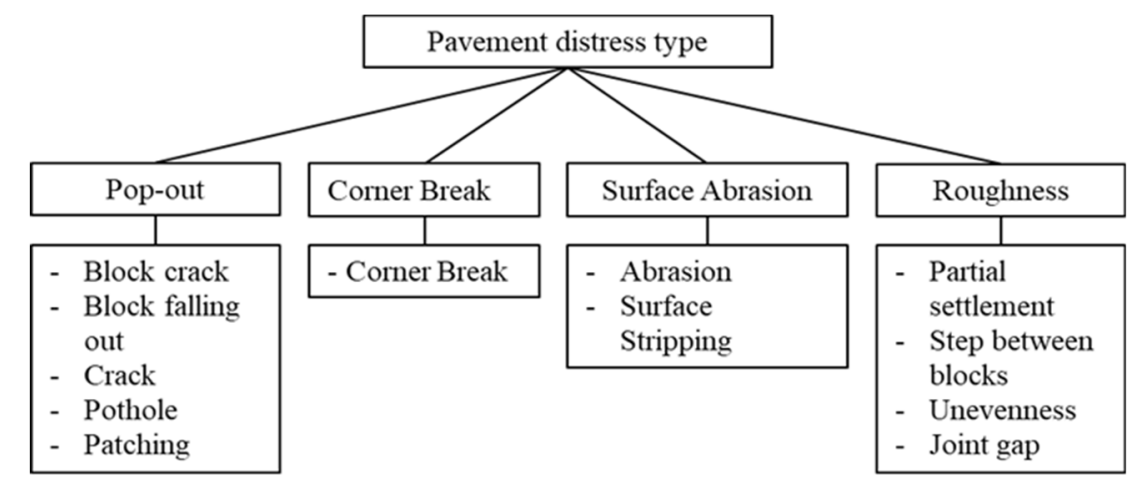

Figure 3. Hierarchy Structure for Analytic Hierarchy Process (AHP).

\subsection{Investigation}

The questionnaire was prepared to investigate the weight among the variables derived from the hierarchy structure. The scale [9] used in the questionnaire is based on Table 3, and the data for pairwise comparisons were obtained from the questionnaire.

Table 3. Saaty's Scale [9].

\begin{tabular}{cc}
\hline Intensity of Importance & Definition \\
\hline $\mathbf{1}$ & Equal Importance \\
\hline 3 & Marginally Strong \\
\hline $\mathbf{5}$ & Strong Importance \\
\hline $\mathbf{7}$ & Very Strong \\
\hline $\mathbf{9}$ & Extremely Strong \\
\hline $\mathbf{2 , 4 , 6 , 8}$ & When Compromise is Needed \\
\hline
\end{tabular}

The questionnaire survey for AHP analysis was based on the responses of 31 civil service officers, who worked on the maintenance and management of the sidewalk. It was conducted at 
the same time and place in order to secure effective questionnaire results. They were thoroughly instructed on the purpose of the questionnaire survey, proposal for applying the developing index, and cautions. Additionally, image data of distress types were shown on a screen during the questionnaire survey. Assuming only one distress at one area, images of similar distress area and distress level were shown to the officers, who then recorded the importance of the two distresses. Particularly, one attractive characteristic of this questionnaire survey is that the government officers, who responded to questionnaires, mainly worked on sidewalk management, therefore, they had a good understanding of the questionnaire contents and distress forms of the sidewalk. Accordingly, an effective result could be obtained.

\subsection{Pairwise Comparisons}

This study carried out pairwise comparisons to compute the weight of each pavement distress based on the results of the questionnaire on importance of each pavement distress type. Pairwise comparisons is a technique to quantify the relative importance (weight) between the components based on the survey results. It applies a mathematical technique to the binary comparison matrix so that decision-making elements can be compared by the hierarchy. First of all, element of the matrix, $a_{i j}$, computes how much the element $i$ is preferable to the element $j$ for the $n$ elements. Then, the comparison matrix $\mathrm{A}=\left(a_{i j}\right) ; i j=1,2, \ldots, n$ can be obtained as Equation (2):

$$
\mathrm{A}=\left[\begin{array}{ccc}
1 & \cdots & a_{1 n} \\
\vdots & 1 & \vdots \\
a_{n 1} & \cdots & 1
\end{array}\right]
$$

When each weight between $n$ number of elements is expressed as $w_{i}(i=1,2, \ldots, n)$, the element, $a_{i j}$, of a comparison matrix $\mathrm{A}$, represents weight rate between row coordinate $i$ and column coordinate $j$. Equation (3) shows the mathematical expression of an element, $a_{i j}$, of a comparison matrix A:

$$
a_{i j}=\frac{w_{i}}{w_{j}}(\text { for } i, j=1,2, \cdots, n) .
$$

Using Equation (3), pairwise comparison matrix of Equation (2) can be expressed as Equation (4):

$$
\mathrm{A}=\left[\begin{array}{ccc}
\frac{w_{1}}{w_{1}} & \cdots & \frac{w_{1}}{w_{n}} \\
\vdots & \ddots & \vdots \\
\frac{w_{n}}{w_{1}} & \cdots & \frac{w_{n}}{w_{n}}
\end{array}\right]
$$

Table 4 shows a pairwise comparison matrix of weights by distress type based on the data collected through the questionnaire survey of this study. The weight of each variable is based on the result of the questionnaire survey. Geometric means were computed to represent importance of each distress type.

Table 4. Comparison Matrix of Weights by Distress Type.

\begin{tabular}{ccccc}
\hline & Pop out & Corner Break & Surface Abrasion & Roughness \\
\hline Pop out & 1 & 7.44 & 3.17 & 2.12 \\
\hline Corner break & 0.13 & 1 & 0.43 & 0.29 \\
\hline Surface abrasion & 0.32 & 2.35 & 1 & 0.67 \\
\hline Roughness & 0.47 & 3.45 & 1.49 & 1 \\
\hline
\end{tabular}

Eigenvector and eigenvalue are calculated to see mathematically whether the pairwise comparison matrix is consistent within an allowable range. Through this computation process, each eigenvector 
and eigenvalue represent weight and inconsistency of the pairwise comparison matrix, respectively. Weights are determined by assuming an eigenvector set for maximum eigenvalue for the matrix. The reason for the importance of maximum eigenvalue is because the larger value indicates less confusion over a decision [10], and its value is given by Equation (5) below:

$$
A_{w}=\lambda_{\max } w
$$

where, $A=$ Pairwise comparison matrix; $w=$ Eigenvector for maximum eigenvalue; and $\lambda_{\max }=$ Maximum eigenvalue.

\subsection{Consistency}

The consistency of the estimated weight can be represented by CI (Consistency Index), as expressed in the following Equation (6):

$$
\mathrm{CI}=\frac{\lambda_{\max }-n}{n-1}
$$

CI indicates the degree of low, random and very logical responses of the questionnaire participants regarding the result of the questionnaire survey, which contradicts the presumption of the weight closing on the true value, and $n$ of Equation (6) represents the number of rows and columns of the pairwise comparison matrix [10]. Generally, if CI exceeds 0.1 , the comparison matrix is deemed to be inconsistent. However, if the dimension of the matrix is only $2 \times 2$, the Saaty's scale may be still useful. However, as the number of dimensions of the matrix increases, the standard needs to be loosened. Accordingly, this study applies the CI more dynamically (lenient) [11]. When the case of changing $\mathrm{CI} \leq 0.1$ to $\mathrm{CI} \leq 0.2$ is considered, the overall $\mathrm{CI}$ did not change significantly. Thus, it follows that setting CI standard to 0.2 is more rational than the case of $\mathrm{CI}$ being 0.1 as the former reflects the opinions of more people (22 vs. 16). CR (Consistency Ratio) was computed using the computed CI and RI (Random Consistency Index). Applying Equation (7) below, the result of Table 5 was obtained. Li et al. (2017) [7] defined RI, which was used to compute CR, as shown in Table 6 below.

$$
\mathrm{CR}=\frac{\mathrm{CI}}{\mathrm{RI}}
$$

CR of this study was computed to be 0.00001 as shown in Table 5 below, and RI values used for the calculation of CR are shown in Table 6. The computed CR indicates very high consistency of the questionnaire data collected in this study and its high validity [7].

Table 5. Computation Result of CR depending on two different CI standards.

\begin{tabular}{ccc}
\hline & $\mathrm{CI} \leq \mathbf{0 . 1}(n=16)$ & $\mathrm{CI} \leq \mathbf{0 . 2}(n=22)$ \\
\hline $\mathrm{CR}$ & 0.00000 & 0.00001 \\
\hline
\end{tabular}

Table 6. RI values.

\begin{tabular}{cccccccccc}
\hline Elements & $\mathbf{1}$ & $\mathbf{2}$ & $\mathbf{3}$ & $\mathbf{4}$ & $\mathbf{5}$ & $\mathbf{6}$ & $\mathbf{7}$ & $\mathbf{8}$ & $\mathbf{9}$ \\
\hline $\mathbf{R I}$ & 0.00 & 0.00 & 0.58 & 0.90 & 1.12 & 1.26 & 1.36 & 1.41 & 1.45 \\
\hline
\end{tabular}

\subsection{Application of Factor Weighting and Modeling}

Table 7 below shows the final result of computing weight of each distress type. The weight was highest for Crack/Loss followed by Roughness, Surface abrasion, and Corner break in descending order. The weight result was then applied to building a model, $y_{1}$. 
Table 7. Factors of Weighting.

\begin{tabular}{cccccc}
\hline Pop out & Roughness & Surface Abrasion & Corner Break & CI & CR \\
\hline 0.521 & 0.244 & 0.164 & 0.070 & 0.00001 & \\
\hline
\end{tabular}

The following modeling, $y_{1}$, (Equation (8)), is initially proposed by setting the maximum SBPCI to be 10 and applying weights of distress types:

$$
y_{1}=10-\left(0.521 \times x_{1}+0.244 \times x_{2}+0.164 \times x_{3}+0.070 \times x_{4}\right)
$$

else, if $y_{1}>0$ or $y_{1}=0$, where, $x_{1}=$ Damage rate of Pop out $(\%) ; x_{2}=$ Damage rate of Roughness $(\%)$; $\mathrm{x}_{3}=$ Damage rate of Surface abrasion $(\%)$; and $\mathrm{x}_{4}=$ Damage rate of Corner break $(\%)$

\section{Development of Regression Model}

A regression analysis of the model $y_{1}$, which was derived from AHP, was used to verify the validity of the model. The data for this model validation were collected from 420 distressed sidewalk sections in Seoul city.

\subsection{Calibration of the Model Scale}

In the case of $y_{1}$, which was developed through AHP, the coefficient of each independent variable is too large to cause the value of the independent variable, $y_{1}$, to be 0 or even a negative number. Therefore, the ratio of each weight for each independent variable was kept the same, but the model scale was calibrated so that a negative index would not be computed. The calibrated values are shown in the following as Equation (9):

$$
y_{2}=10-\left(1 \times 10^{-2} \times x_{1}+4.683 \times 10^{-3} \times x_{2}+3.148 \times 10^{-3} \times x_{3}+1.344 \times 10^{-3} \times x_{4}\right)
$$

where, $\quad x_{1}=$ Damage rate of Pop out $(\%) ; x_{2}=$ Damage rate of Roughness $(\%) ; x_{3}=$ Damage rate of Surface abrasion (\%); and $\mathrm{x}_{4}=$ Damage rate of Corner break $(\%)$.

\subsection{Correlation between Variables}

Pearson's correlation coefficient was computed in order to analyze the correlation between each independent variable and the value of $y_{2}$ as summarized in Table 8 below. The independent variables, $\mathrm{x}_{1}$ (Pop-out), $\mathrm{x}_{2}$ (Roughness), $\mathrm{x}_{3}$ (Surface abrasion), were correlated with $\mathrm{y}_{2}$ in descending order, and they were all statistically significant. However, the case of $\mathrm{x}_{4}$ (Corner break) exhibited a positive correlation with the dependent variable to be excluded from variables of SBPCI. This was explained that the frequency of $\mathrm{x}_{4}$ (Corner break) distress was so low that the distress rate was surveyed to be too low to cause this result.

Table 8. Pearson's correlation coefficient.

\begin{tabular}{ccccc}
\hline \multicolumn{4}{c}{ Pearson's Correlation Coefficient, $\mathrm{N}=420$, H0 : Rho $=0$ Null Hypothesis Prob $>|\mathbf{r}|$} \\
\hline & $x_{\mathbf{1}}$ (Pop out $)$ & $x_{\mathbf{2}}$ (Roughness) & $x_{3}$ (Surface abrasion) & $x_{4}$ (Corner break) \\
\hline $\begin{array}{c}\text { Correlation } \\
\text { coefficient }\end{array}$ & -0.759 & -0.259 & -0.202 & 0.049 \\
\hline$p$-value & $<0.0001$ & $<0.0001$ & $<0.0001$ & 0.319 \\
\hline
\end{tabular}

\subsection{Model Development}

Result of regression analysis of the scale calibrated model $y_{2}$ is shown in the following Equation (10) with Tables 9 and 10. It shows the $p$-values for all variables were less than 0.05 and no multicollinearity 
was observed. $R^{2}$ was about 0.99 , meaning that the regression model is reliable and valid by reflecting a significant part of AHP results of experts' opinions.

$$
\mathrm{SBPCI}=9.994-0.099 * \operatorname{Po}(\%)-0.047 * \operatorname{Ro}(\%)-0.031 * \mathrm{Sa}(\%)
$$

where, Po $=$ Damage rate of Pop out $(\%)$

Ro $=$ Damage rate of Roughness $(\%)$

$\mathrm{Sa}=$ Damage rate of Surface abrasion $(\%)$

Table 9. Technical statistics of regression model.

\begin{tabular}{cccccc}
\hline \multicolumn{6}{l}{ Number of Observations Read/Used } \\
\hline \multicolumn{7}{c}{420} \\
\hline Source & DF & Sum of Squares & Mean Square & F Value & Pr > F \\
\hline Model & 3 & 675.119 & 225.040 & 226255 & $<0.0001$ \\
\hline Error & 416 & 0.414 & 0.000 & & \\
\hline Corrected Total & 419 & 675.533 & & & \\
\hline
\end{tabular}

Table 10. Regression analysis result.

\begin{tabular}{cccccc}
\hline Variable & Parameter Estimate & Standard Error & Type II SS & F Value & Pr $>$ F \\
\hline Intercept & 9.994 & 0.003 & 15913 & $1.6 \times 10^{7}$ & $<0.0001$ \\
\hline Pop out & -0.099 & 0.000 & 587.504 & 590676 & $<0.0001$ \\
\hline Roughness & -0.047 & 0.000 & 234.253 & 235518 & $<0.0001$ \\
\hline Surface abrasion & -0.031 & 0.000 & 105.408 & 105977 & $<0.0001$ \\
\hline
\end{tabular}

\subsection{Validity Analysis of the Model}

As shown in Figure 4, residual analysis was performed using scatter plot (Pop out, Roughness, Surface abrasion from upper left corner) in order to evaluate the applicability of the SBPCI model developed in this study, and to determine the heterogeneity of the residuals and the necessity of the secondary term. As a result of the analysis, it was found that homoscedasticity was observed in all the variables and the variable conversion was not necessary. 

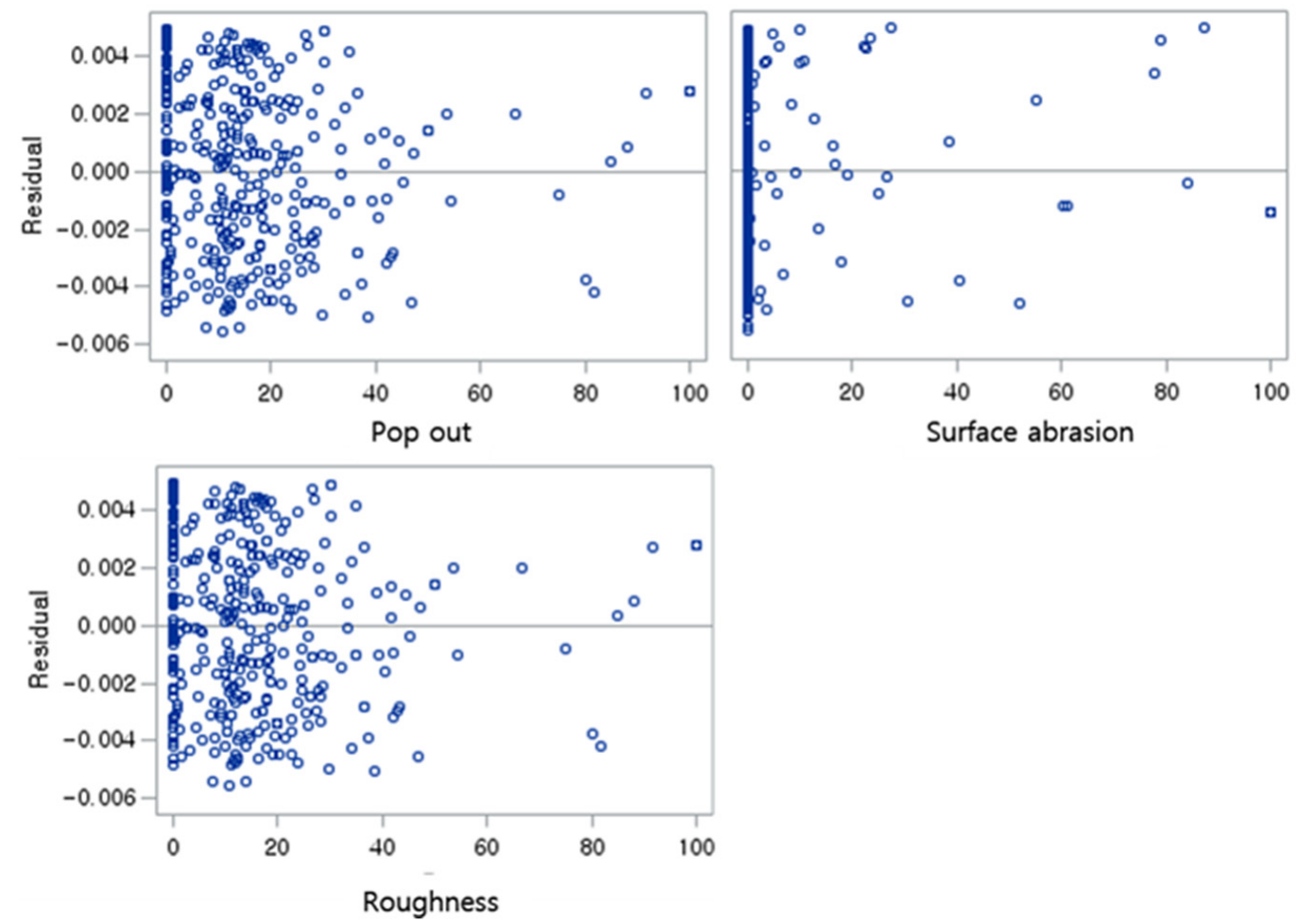

Figure 4. Distribution of residuals for each dependent variable.

\subsection{Sensitivity Analysis of the SBPCI Model Equation}

Sensitivity analysis was conducted on the 420 sidewalk pavement sections, where actual distress occurred, to investigate the effect of each independent variable on the SBPCI. For the sensitivity analysis of three independent variables, the severity by distress level was classified as L (Low), M (Medium) and $\mathrm{H}$ (High). Medium value used arithmetic average, and the boundary for low and high level of severity was set to be upper and lower 20 percentiles, respectively. The boundary values are shown in Table 11 below.

Table 11. Average severity of each distress.

\begin{tabular}{cccc}
\hline & Pop out & Roughness & Surface Abrasion \\
\hline Average & 5.43 & 15.41 & 4.08 \\
\hline Low & 0.00 & 0.00 & 0.00 \\
\hline Medium & 5.43 & 15.41 & 4.08 \\
\hline High & 18.05 & 32.64 & 20.50 \\
\hline
\end{tabular}

Sensitivity analysis used the method of substituting the value of the severity of each independent variable and the average value of the remaining variables into the SBPCI model equation. Sensitivity was compared through the slope of each independent variable. The greater the slope, the greater the effect on SBPCI. The impact of three independent variables on SBPCI was in the descending order of Roughness, Pop out, and Surface abrasion at the low and medium severity range. It was Pop out, Roughness, and Surface abrasion in descending order at the medium and high severity range. Sensitivity analysis results are shown in Figure 5. 


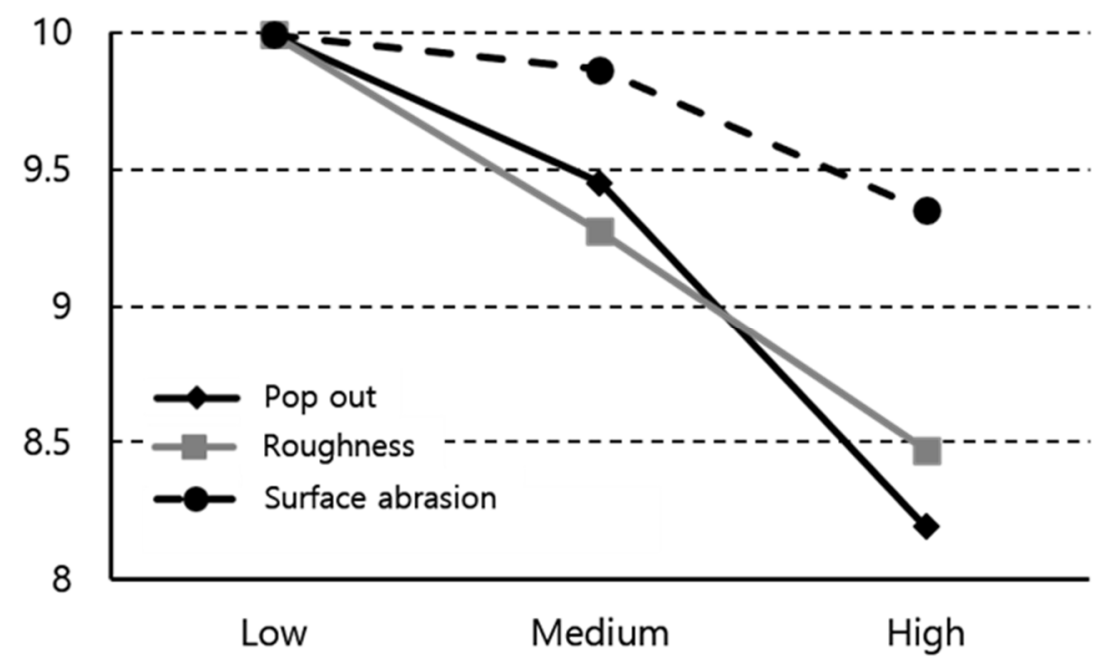

Figure 5. Sensitivity analysis results by distress.

\section{Applicability}

Table 12 shows the level of service according to the current sidewalk pavement condition in Korea [12], and it is deemed that the repair is required from ' $\mathrm{D}$ ' grade. ' $\mathrm{D}$ ' rating means a pavement condition that affects the normal traffic of pedestrians and where the transportation is weak. It signifies the distress of the sidewalk pavement in the unit section is more than $50 \%$.

Table 12. Service level of sidewalk pavement condition in Korea [13].

\begin{tabular}{ccc}
\hline Rating & Service Level of the Pavement Condition & Distress Rate \\
\hline A (Very Good) & Pavement condition as new or new & \\
\hline B (Good) & $\begin{array}{c}\text { The sidewalk pavement is in a uniform state although it is not smooth } \\
\text { as a whole }\end{array}$ & \\
\hline C (Fair) & $\begin{array}{c}\text { The sidewalk pavement condition is such that consideration for repair } \\
\text { is required depending on the aging of the pavement section }\end{array}$ & $50 \%$ or above \\
\hline D (Poor) & Pavement condition to affect traffic & $75 \%$ or above \\
\hline E (Very Poor) & State of pavement inaccessible for normal traffic &
\end{tabular}

Currently, no quantitative criteria are set in the maintenance decision for the sidewalk pavement and only the qualitative criteria through visual inspection are investigated as shown in Table 12. Therefore, it was necessary to determine the SBPCI standard score, that requires maintenance, in order to apply the SBPCI developed in this study to practice. Accordingly, the score was developed through the T-test between the sections, that were considered to require maintenance based on the distress rate of above 50\%; and the sections, where maintenance was considered necessary based on the SBPCI score. As a result, when SBPCI 6.6 was used as the reference score, it was statistically the same as the repair section of $50 \%$ or more distress rate. It was also determined that the derived number of SBPCI 6.6 could be used by the working group performing the maintenance as a quantitative threshold to determine the necessity of maintenance.

In order to prove that the section selected through distress rate and the section selected through SBPCI were not statistically different, the section with distress rate of $50 \%$ or more was classified as 'group 1', and the section with SBPCI less than 6.6 was classified as 'group 2'. $p$-values of the T-test results between the two groups was 0.069 . Thus, in the null hypotheses, there is no difference between 'group 1' and 'group 2', and cannot be rejected, meaning that there is no difference between the two groups (Table 13). Table 14 below summarizes the statistical test result. 
Table 13. T-test group statistic between two groups.

\begin{tabular}{ccccc}
\hline Group & $\mathbf{N}$ & Avg. & Standard Deviation & Average of Standard Error \\
\hline $\mathbf{1}$ & 420 & 0.093 & 0.291 & 0.014 \\
\hline $\mathbf{2}$ & 420 & 0.060 & 0.237 & 0.012 \\
\hline
\end{tabular}

Table 14. Independent sample test between two groups.

\begin{tabular}{ccccccccc}
\hline $\begin{array}{c}\text { Levene } \\
\text { Homogeneity of } \\
\text { Variance Test }\end{array}$ & & T-Test of Comparison of Means for Difference & \multicolumn{2}{c}{$\begin{array}{c}95 \% \text { Confidence } \\
\text { Interval }\end{array}$} \\
\hline F & $p$-Value & $\mathrm{T}$ & $\begin{array}{c}\text { Degrees of } \\
\text { Freedom }\end{array}$ & $p$-Value & $\begin{array}{c}\text { Mean } \\
\text { Difference }\end{array}$ & $\begin{array}{c}\text { Standard Error of } \\
\text { Estimate }\end{array}$ & $\begin{array}{c}\text { Lower } \\
\text { Bound }\end{array}$ & $\begin{array}{c}\text { Upper } \\
\text { Bound }\end{array}$ \\
\hline 13.474 & 0.000 & 1.822 & 838 & 0.069 & 0.033 & 0.018 & -0.003 & 0.069 \\
\hline
\end{tabular}

\section{Future Work}

The model presented in this study is in need of further research on the quantification of the size of raggedness and pedestrian satisfaction factor improvement. For this part, application studies with advanced sensors such as "RGB-D Sensor" or "3D Scanner" [12] will be required. Additionally, easier access to SBPCI ratings is needed, to help readers better understand and determine the implementation of service and maintenance based on the ratings.

\section{Conclusions}

The purpose of this study was to develop an evaluation index that can quantify the pavement condition of sidewalks in Korea. Sidewalk Block Pavement Condition Index (SBPCI) was developed through a multiple decision-making system, AHP (Analytical Hierarchy Process), which quantified the qualitative criteria of the sidewalk pavement managers. The results of this study are summarized as follows.

The distress types of the sidewalk pavement were classified into four types, Pop out, Roughness, Surface abrasion and Corner break.

Based on the results of the questionnaire survey conducted by the sidewalk pavement practitioners, AHP was used in this study to quantify the condition of the sidewalk pavement by calculating the weight for the distress types.

AHP analysis of the weights for each type of distress showed Pop out (0.521), Roughness (0.244), Surface abrasion (0.164), and Corner break (0.070) in descending order.

As a result of analyzing the correlation between each independent variable, the correlation among Pop out, Roughness, and Surface abrasion was found to be significant. However, Corner break was less correlated with the actual distress rate and occurred less frequently.

A regression analysis was carried out to establish a pavement condition index for the sidewalk pavement using the weight values calculated by AHP. $R^{2}$ of the model derived from this study was calculated as 0.99 with $p$-value less than 0.05 , indicating a statistically significance of the model.

Sensitivity of Roughness, Pop out and Surface abrasion was found to be in descending order at low and medium severity range. It was Pop out, Roughness, and Surface abrasion in descending order at the medium and high severity range. This means that it is necessary to focus on specific distress during actual management of the sidewalk pavement, since Roughness and Pop out had a great influence on the SBPCI in good and bad pavement conditions, respectively.

It was found that the distress rate of $50 \%$, which is the current standard for repairing the sidewalk pavement, equated to be about 6.6 points of SBPCI. For this, T-test was performed on each selected repair section through the distress rate and SBPCI. As a result, the $p$-value was 0.069 , and the difference between the two groups was not statistically significant since the existing null hypothesis could not be rejected. 
Author Contributions: Conceptualization, J.K. and D.P.; methodology, J.K. and D.J.; software, D.J.; validation, J.K. and Y.S.; formal analysis, D.J.; investigation, J.K. and D.P.; resources, J.K. and D.P.; data curation, J.K. and D.J.; writing—original draft preparation, D.J.; writing—review and editing, J.K. and Y.S.; visualization, D.J.; supervision, J.K. and D.P.; project administration, J.K.

Funding: This research received no external funding.

Conflicts of Interest: The authors declare no conflicts of interest.

\section{References}

1. Feereira, A.; Luis, P.; Antunes, A. A segment-linked optimization model for deterministic pavement management systems. Int. J. Pavement Eng. 2002, 3. [CrossRef]

2. Cottrell, W.D.; Bryan, S.; Chilukuri, B.R.; Kalyani, V. Transportation Infrastructure maintenance management case study of a small urban city. J. Infrastruct Syst. 2009, 15, 120-132. [CrossRef]

3. Oakland, Citywide Sidewalk Condition and ADA Survey. Final Report. TranSystems: Oakland, CA, USA, 2007. Available online: www2.oaklandnet.com/OAK036290 (accessed on 11 December 2019).

4. Amoroso, S.; Caruso, L. Percorsi pedonali e attraversamenti stradali: elementi per lo studio della qualita e della sicurezza. In Proceedings of the 3rd National Congress of Road City Safety, Lucca, Italy, 21 February 2008.

5. Corazza, M.V.; Mascio, P.D.; Moretti, L. Managing sidewalk pavement maintenance: A case study to increase pedestrian safety. J. Traffic Transp. Eng. 2016, 3, 203-214. [CrossRef]

6. Transport for London Highway Asset Management Survey-Footways, Final Report. 2011. Available online: http://content.tfl.gov.uk/highway-asset-management-survey-footways-report.pdf (accessed on 11 December 2019).

7. Ahmed, S.; Vedagiri, P.; Krishna, K.V. Prioritization of pavement maintenance sections using objective based Analytic Hierarchy Process. Int. J. Pavement Res. Technol. 2017, 10, 158-170. [CrossRef]

8. Li, H.; Ni, F.; Dong, Q.; Zhu, Y. Application of analytic hierarchy process in network level pavement maintenance decision-making. Int. J. Pavement Res. Technol. 2017, 11, 1-10. [CrossRef]

9. Saaty, T.L.; Vargas, L.G. The Logic of Priorities: Application in Business, Energy; Health, and Tranportation, Kluwer/Nijhoff Publishing: Springer: Boston, MA, USA, 1982.

10. Yang, C. Developing Decision-Making Process for Prioritizing Potential Alternatives of Truck Management Strategies. Ph.D. Thesis, University of California, Irvine, CA, USA, 2008.

11. TxDOT. Project Development Process Manual, Final Report. 2014. Available online: http://ceprofs.civil.tamu. edu/dlord/CVEN_456_Course_Material/pdp.pdf (accessed on 11 December 2019).

12. Ahmadreza, M.; Amir, G.; Mohammad, R.J.; Sayna, F.Y. Estimating Pavement Roughness by Fusing Color and Depth Data Obtained form an Inexpensive RGB-D Sensor. Sensors 2019, 19, 1655. [CrossRef]

13. Ministry of Land, Infrastructure and Transport. Guidelines for Sidewalk Construction and Management. Available online: http://molit.go.kr/USR/I0204/m_45/dtl.jsp?idx=15594 (accessed on 11 December 2019).

(C) 2019 by the authors. Licensee MDPI, Basel, Switzerland. This article is an open access article distributed under the terms and conditions of the Creative Commons Attribution (CC BY) license (http://creativecommons.org/licenses/by/4.0/). 